\title{
A multimodal approach for simultaneous mass and rotary inertia sensing from vibrating cantilevers
}

\author{
S. Adhikari ${ }^{\mathrm{a}, *}$, H. H. Khodaparast ${ }^{\mathrm{a}}$ \\ ${ }^{a}$ College of Engineering, Swansea University, Swansea, UK
}

\begin{abstract}
Nano and micromechanical mass sensing using cantilever oscillators of different lengthscales has been an established approach. The main principle underpinning this technique is the shift in the resonance frequency caused by the additional mass in the dynamic system. While the mass of an object to be sensed is useful information, some idea about the shape of the object would be an additional benefit. The shape information may be used to make a distinction between two different objects of the same mass. This paper establishes the conceptual framework for simultaneous sensing of the mass as well as the rotary inertia of an object attached to a vibrating cantilever beam. The rotary inertia of an object gives additional insight into its shape, which is a key motivation of this work. It is shown that by using two modes it is possible to formulate two coupled nonlinear equations, which in turn can be solved to obtain the mass and the rotary inertia simultaneously from the frequency shifts of first two vibration modes. Euler-Bernoulli beam theory and an energy approach are used to derive closed-form expressions for the identified mass and rotary inertia from the measured frequency shifts. Analytical expressions are validated using high fidelity finite element simulation results.
\end{abstract}

Keywords: Nanomechanical sensor; frequency shift; mass sensing; rotary inertia; cantilever beam

\section{Introduction}

Nano-mechanical sensing has established itself as a promising research direction and generated significant interest across the research community over the past decade. In particular vibrating nano-mechanical cantilevers have received wide attention due to the possibility of obtaining resonance frequency very accurately. Consequently, sensing of an attached mass to a cantilever sensor by exploiting a frequency-shift has emerged as a powerful approach [1-10]. This paper is aimed at investigating the possibility of sensing mass as well as rotary inertia of an attached object. The magnitude of the mass gives the basic information of an attached object. But it gives no information about the shape and size of such objects. Rotary inertia can give some further insights into its shape and size.

\footnotetext{
*Corresponding author. Tel: +44 (0)1792 602088, Fax: + 44 (0)1792 295676

Email addresses: S.Adhikari@swansea.ac.uk (S. Adhikari),

H.Haddadkhodaparast@swansea.ac.uk (H. H. Khodaparast)
} 
This paper proposes a novel way by which both the mass and rotatory inertia of an object can be obtained simultaneously from frequency shifts. With the additional information of the rotatory inertia, it may be possible to infer more about the attached object to a cantilever nanosensor, which is a key motivation for this work.

Nano-scale sensors [4, 11-14] play crucial roles in environmental monitoring (e.g., detection of gas) [15], chemical process control [16] and biomedical applications [17$22]$. Among various nano-devices, Carbon nanotubes (CNTs) have been used as electrochemical sensing system [23-26], nanosensors [13, 14, 27-29] and nanoactuators [30]. The use of a CNT to make the lightest inertial balance is essentially to make a ultrasensitive nano-scale mass spectrometer [19, 20], electroanalytical nanotube devices [21, 31] and electro-mechanical actuators for artificial muscles [32]. Nano-bio sensors [33] are being developed on the fact that biological fragments can be immobilized either in the hollow cavity or on the surface of carbon nanotubes $[17,18]$. Building and designing such nanosensors that is able to make measurements of external deposited agents [32, 34-36] with ultrahigh resolution $[14,37]$ is one of the main goals in the field of nano mechanical sensing and is the topic of this paper.

The main scientific challenge to identify rotatory inertia is the requirement of an extra equation in addition to what we know for mass identification. This is realised by using the second mode of vibration of the cantilever. It is shown that by using two modes it is possible to formulate two coupled nonlinear equations, which in turn can be solved to obtain mass and rotary inertia simultaneously from the frequency shifts of the first two vibration modes. Closed-form expressions based on the kinetic and potential energy of a cantilever is proposed. Analytical results are validated using high fidelity molecular mechanics simulation. This theoretical study may motivate experimentalist attending the conference and can pave the way of further collaboration.

Outline of the paper is as follows. In Section 2, the equation of motion and relevant boundary conditions are discussed for the dynamic analysis of nano-cantilevers with attached mass. The energy approach to derive the resonance frequencies of the cantilevers taking the rotary-inertia effect are derived in Section 3. In Section 4, the closed-form expressions to identify the mass and rotary-inertia of the attached object from the frequencyshifts are derived. In Section 5 the proposed methodology is applied to a single-walled carbon nanotube (SWCNT) in cantilever configuration for illustration. Derived closedform expressions are numerically validated with detailed finite element results. Finally, in Section 6 some conclusions are drawn based on the results obtained in the paper.

\section{Dynamics of nano-cantilevers with attached mass}

\subsection{Equation of motion and boundary conditions}

Euler-Bernoulli beam bending theory [38] is used to model the dynamics of the nanocantilevers. In Fig. 1 an example of a carbon nano-tube resonator with attached mass (DeOxy Thymidine) is shown. Mathematical idealisation by a uniform cantilever beam with a tip mass is also shown in the figure. The equation of motion of free-vibration can be expressed as

$$
E I \frac{\partial^{4} y(x, t)}{\partial x^{4}}+\rho A \frac{\partial^{2} y(x, t)}{\partial t^{2}}=0
$$




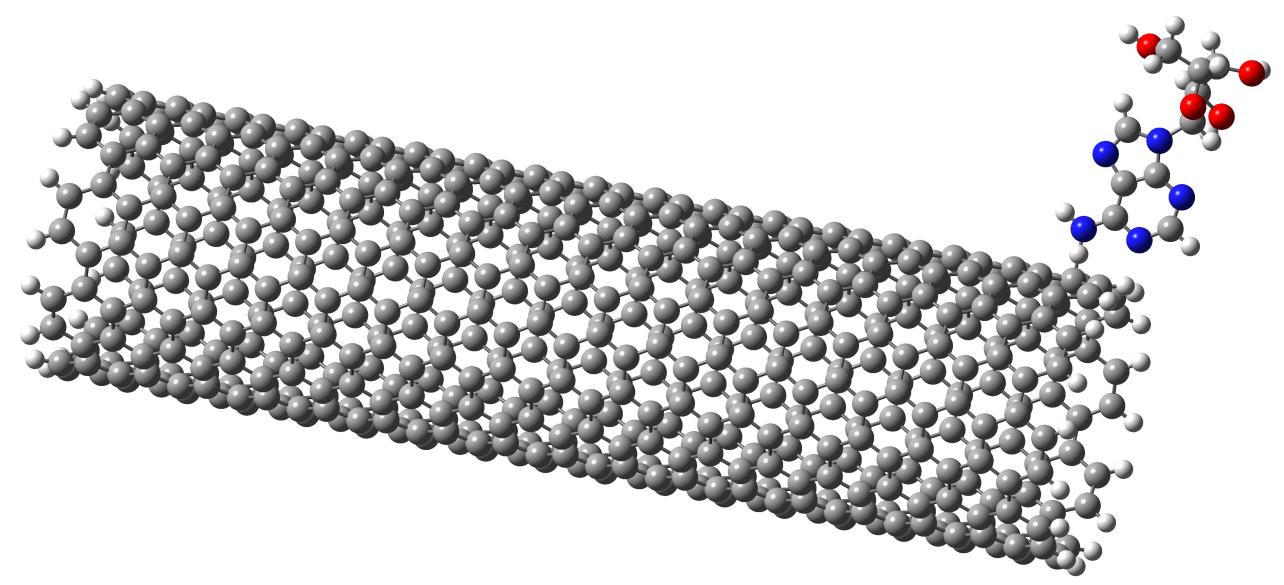

(a) Single wall carbon nanotube with a tip mass

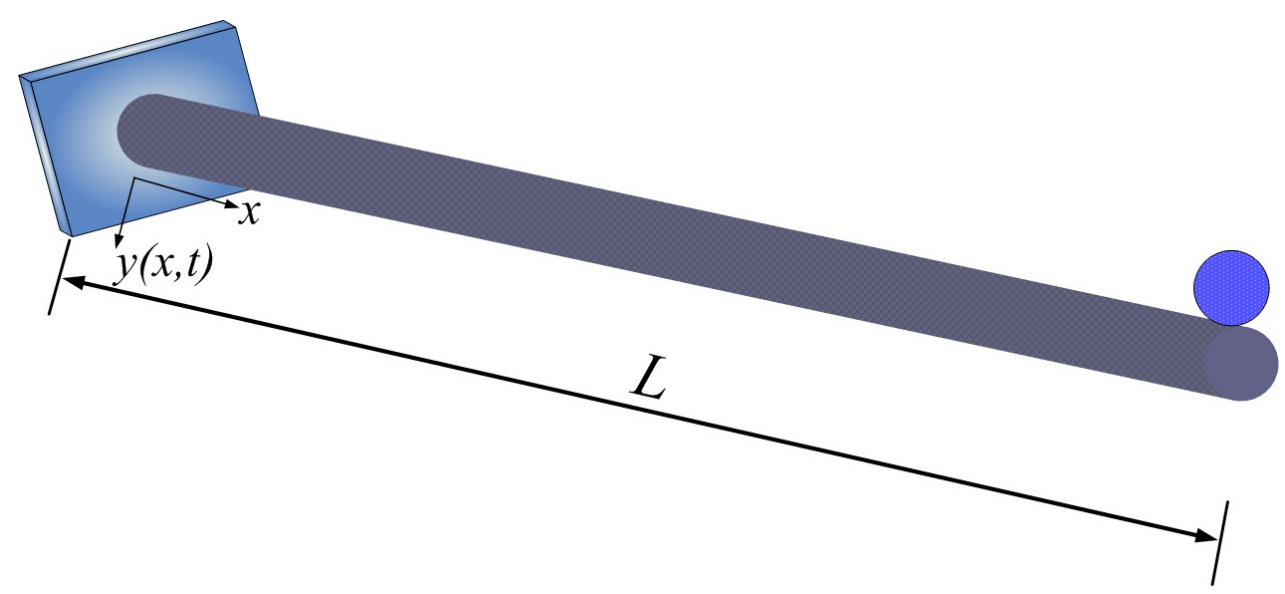

(b) Mathematical idealization of (a): point mass at the tip

Fig. 1: A cantilevered carbon nanotube resonator with attached mass. The inertia effect arises from 'height' of the attached object (DeOxy Thymidine used as an example). (a) Original configuration with a point mass at the tip; (b) Mathematical idealisation with a point mass at the tip.

where $x$ is the coordinate along the length of the cantilever oscillator, $t$ is the time, $y(x, t)$ is the transverse displacement of the cantilever oscillator, $E$ is the Young's modulus, $I$ is the second-moment of the cross-sectional area $A$ and $\rho$ is the density of the material. Suppose the length of the cantilever oscillator is $L$. The effect of distributed rotary inertia and shear deformation has been neglected in equation (1). These assumption is likely to be valid for long and thin cantilever resonators.

For the cantilevered oscillator without any attached mass, the resonance frequencies can be obtained [39] from

$$
f_{0_{j}}=\frac{c_{0}}{2 \pi} \lambda_{j}^{2}
$$

where $c_{0}$ is a parameter depending on the material and geometric properties of the cantilever as

$$
c_{0}=\sqrt{\frac{E I}{\rho A L^{4}}}
$$


For a carbon nanotube with diameter $D$ and thickness $t$ (note that $t<<D$ ) the crosssectional area $A$ and area moment of inertia $I$ can be obtained as $A \approx \pi D t$ and $I \approx \frac{\pi}{8} D^{3} t$. Using these we can obtain

$$
c_{0} \approx \sqrt{\frac{E \frac{\pi}{8} D^{3} t}{\rho \pi D t L^{4}}}=\frac{1}{2} \frac{D}{L} \sqrt{\frac{E}{2 \rho L^{2}}}
$$

The constants $\lambda_{j}$ should be obtained by [40] solving the following transcendental equation

$$
\cos \lambda \cosh \lambda+1=0
$$

The vibration mode shape can be expressed as

$$
Y_{j}(\xi)=\left(\cosh \lambda_{j} \xi-\cos \lambda_{j} \xi\right)-\left(\frac{\sinh \lambda_{j}-\sin \lambda_{j}}{\cosh \lambda_{j}+\cos \lambda_{j}}\right)\left(\sinh \lambda_{j} \xi-\sin \lambda_{j} \xi\right)
$$

where

$$
\xi=\frac{x}{L}
$$

is the normalised coordinate along the length of the cantilever oscillator. The values of $\lambda$ arising from the solution of equation (5) are be given by $\lambda_{1}=1.8751, \lambda_{2}=4.6941$, $\lambda_{3}=7.8547, \lambda_{4}=10.9954$ and $\lambda_{5}=14.1371$. For $j>5$, in general [40] $\lambda_{j}=(2 j-1) \pi / 2$. For sensing applications, we are interested in the first few modes of vibration only. In this paper the first two modes of vibration will be used. The analysis based on the Euler-Bernoulli beam bending theory is well known and further details are available in established texts such as [39]. Conventionally an added mass is not considered in the classical Euler-Bernoulli theory. However, such effects can be incorporated in various ways, see for example [41]. We employ the analytical techniques exposed in the existing literature to consider the effect of mass and rotary inertia simultaneously within the scope of classical Euler-Bernoulli beam bending framework.

The equation of motion of the cantilever resonator with the attached mass including the rotary inertia effect can be expressed by the same equation as (1). However, the boundary conditions must be changed to include the additional effects. Consider that the attached object as depicted in Fig. 1(b) has mass $M$ and rotary inertia $J$. The four boundary conditions associated with equation (1) can be expressed as

- Deflection at $x=0$ :

$$
y(0, t)=0
$$

- Slope at $x=0$ :

$$
\frac{\partial y(x, t)}{\partial x}=0
$$

- Bending moment at $x=L$ :

$$
E I \frac{\partial^{2} y(x, t)}{\partial x^{2}}+J \frac{\partial \ddot{y}(x, t)}{\partial x}=\left.0\right|_{x=L}
$$


- Shear force at $x=L$ :

$$
E I \frac{\partial^{3} y(x, t)}{\partial x^{3}}-M \ddot{y}(x, t)=\left.0\right|_{x=L}
$$

Here $(\bullet)$ denotes derivative with respective to $t$. Assuming harmonic solution we have

$$
y(x, t)=Y(\xi) \exp [\mathrm{i} \omega t]
$$

where $i$ is the unit imaginary number $i=\sqrt{-1}$ and $\omega$ is the frequency. Substituting this in the equation of motion and the boundary conditions and after some simplification we have

$$
\begin{aligned}
& \frac{\partial^{4} Y(\xi)}{\partial \xi^{4}}-\Omega^{2} Y(\xi)=0 \\
& Y(0)=0, Y^{\prime}(0)=0, Y^{\prime \prime}(1)-\beta \Omega^{2} Y^{\prime}(1)=0 \quad \text { and } \quad Y^{\prime \prime \prime}(1)+\alpha \Omega^{2} Y(1)=0
\end{aligned}
$$

Here $(\bullet)^{\prime}$ denotes derivative with respective to $\xi$ and

$$
\begin{array}{rlrl}
\Omega^{2} & =\omega^{2} / c_{0}^{2} & \text { (nondimensional frequency parameter) } \\
\alpha & =\frac{M}{\rho A L} \quad \text { (mass ratio) } \\
\text { and } \quad \beta & =\frac{J}{\rho A L^{3}} \quad \text { (inertia ratio) }
\end{array}
$$

The mass ratio $\alpha$ is an independent parameter which only depends on the mass (volume $x$ density) of the attached object. However, the inertia ratio $\beta$ is in general will not be an independent quantity as it is likely to depend on the mass of the object. For standard geometric shapes, tables for volume, area and moment of inertia are available [42]. In general we can express the rotary inertia as $J=M r^{2}$ where $r$ is an equivalent length parameter. Examples include, a rectangle with height $h, r^{2}=h^{2} / 3$; a hollow cylinder with inner and outer radius $R_{1}$ and $R_{2}, r^{2}=\left(R_{1}^{2}+R_{2}^{2}\right) / 2$; a solid sphere with radius $R$, $r^{2}=2 R^{2} / 5$; a thinwalled hollow sphere with radius $R, r^{2}=2 R^{2} / 3$. Using the definitions of $\alpha$ and $\beta$, we can easily derive that

$$
\beta=\alpha\left(\frac{r}{L}\right)^{2}
$$

As $r$ in the above equation is different for different shapes, identifying $\alpha$ and then $\beta$ can give an indication of the shape of the object to be sensed. Although $\alpha$ and $\beta$ are not completely independent, for mathematical convenience we consider them independent and aim to determine both of them simultaneously from frequency-shifts. To achieve this, we first need to obtain the natural frequencies of the system, which is discussed in the next subsection. 


\subsection{Frequency equation}

Natural frequencies of the system can be obtained from the free vibration problem. We refer to [39] for further discussions to free vibration analysis of distributed parameter systems. Assuming a solution of the exponential form

$$
Y(\xi)=\exp \{\eta \xi\}
$$

and substituting in the equation of motion (13) results

$$
\eta^{4}-\Omega^{2}=0
$$

Using the definition of $\lambda$ consistent with Eq. (2) we have

$$
\lambda^{2}=\Omega
$$

Using the this relationship, the four solutions of $\eta$ in Eq. (20) can be expresses as

$$
\eta^{4}-\lambda^{4}=0 \quad \text { or } \quad \eta= \pm \mathrm{i} \lambda, \quad \pm \lambda
$$

In view of the solutions in Eq. (22), the displacement field with the beam can be expressed by linear combination of the basic functions $e^{-\mathrm{i} \lambda \xi}, e^{\mathrm{i} \lambda \xi}, e^{\lambda \xi}$ and $e^{-\lambda \xi}$ so that the vector of the basic functions is given by $\mathbf{s}(\xi)=\left\{e^{-\mathrm{i} \lambda \xi}, e^{\mathrm{i} \lambda \xi}, e^{\lambda \xi}, e^{-\lambda \xi}\right\}^{T}$. We can also express $\mathbf{s}(\xi)$ in terms of trigonometric functions. Considering $e^{ \pm \mathrm{i} \lambda \xi}=\cos (\lambda \xi) \pm \mathrm{i} \sin (\lambda \xi)$ and $e^{ \pm \lambda \xi}=\cosh (\lambda x) \pm \mathrm{i} \sinh (\lambda \xi)$, the vector $\mathbf{s}(\xi)$ can be alternatively represented as

$$
\mathbf{s}(\xi)=\left\{\begin{array}{c}
\sin (\lambda \xi) \\
\cos (\lambda \xi) \\
\sinh (\lambda \xi) \\
\cosh (\lambda \xi)
\end{array}\right\}
$$

The displacement field $Y(\xi)$ therefore can be expressed as a linear combination

$$
\begin{aligned}
Y(\xi) & =a_{1} \sin \lambda \xi+a_{2} \cos \lambda \xi+a_{3} \sinh \lambda \xi+a_{4} \cosh \lambda \xi \\
\text { or } \quad Y(\xi) & =\mathbf{s}^{T}(\xi) \mathbf{a}
\end{aligned}
$$

where the vector

$$
\mathbf{a}=\left\{a_{1}, a_{2}, a_{3}, a_{4}\right\}
$$

Applying the boundary conditions in equation (14) on the expression of $Y(\xi)$ in $(24)$ we have

$$
\mathbf{R a}=\mathbf{0}
$$

where the matrix

$$
\begin{aligned}
& \mathbf{R}=\mathbf{c} \\
& {\left[\begin{array}{cccc}
0 & 1 & 0 & 1 \\
\lambda & 0 & \lambda & 0 \\
-\sin (\lambda) \lambda^{2}-\lambda^{5} \beta \cos (\lambda) & -\cos (\lambda) \lambda^{2}+\lambda^{5} \beta \sin (\lambda) & \sinh (\lambda) \lambda^{2}-\lambda^{5} \beta \cosh (\lambda) & \cosh (\lambda) \lambda^{2}-\lambda^{5} \beta \sinh (\lambda) \\
-\cos (\lambda) \lambda^{3}+\lambda^{4} \alpha \sin (\lambda) & \sin (\lambda) \lambda^{3}+\lambda^{4} \alpha \cos (\lambda) & \cosh (\lambda) \lambda^{3}+\lambda^{4} \alpha \sinh (\lambda) & \sinh (\lambda) \lambda^{3}+\lambda^{4} \alpha \cosh (\lambda)
\end{array}\right]}
\end{aligned}
$$


The constant vector in equation (26) cannot be zero. Therefore, the equation governing the natural frequencies is given by

$$
\operatorname{det}\{\mathbf{R}\}=0
$$

which upon simplifying results into

$$
\begin{aligned}
& \left((1-\cos (\lambda) \cosh (\lambda)) \lambda^{3} \beta-\sin (\lambda) \cosh (\lambda)+\cos (\lambda) \sinh (\lambda)\right) \lambda \alpha \\
& \quad-(\cos (\lambda) \sinh (\lambda)+\sin (\lambda) \cosh (\lambda)) \lambda^{3} \beta+[\cos (\lambda) \cosh (\lambda)+1]=0
\end{aligned}
$$

In the special case when the mass and the inertia of the attached particle is ignored, substituting $\alpha=\beta=0$, equation (29) reduces to the classical equation (5). If the inertia of the attached particle is ignored substituting $\beta=0$, equation (29) reduces to the equation of a cantilever with an attached tip mass (see for example [8]) as

$$
(-\sin (\lambda) \cosh (\lambda)+\cos (\lambda) \sinh (\lambda)) \lambda \alpha+[\cos (\lambda) \cosh (\lambda)+1]=0
$$

The natural frequencies can be obtained by solving the transcendental equation (29) for $\lambda$ and substituting those in equation (2). Due to the nonlinearity of this transcendental equation, it needs to be solved numerically.

\section{Energy approach for vibrational frequencies}

The frequency equation (29) in the previous section is obtained by considering the differential equation and the boundary conditions in an exact manner. This equation is complex enough so that a simple relationship between the change in the mass and rotary inertia and the shift in the frequency is not available. As we have two unknowns $\alpha$ and $\beta$, two frequency shifts are necessary to identify them. In this section, we develop a new energy-based approach to obtain these quantities.

An arbitrary $j$-th natural frequency of a cantilever oscillator can be expressed as

$$
f_{j}=\frac{1}{2 \pi} \sqrt{\frac{k_{e q_{j}}}{m_{e q_{j}}}}, j=1,2,3 \cdots
$$

Here $k_{e q_{j}}$ and $m_{e q_{j}}$ are respectively equivalent stiffness and mass of the cantilever oscillator in the $j$-th mode of vibration. The equivalent mass $m_{e q_{j}}$ changes depending on the mass and inertia of the attached object. Suppose $Y_{j}$ is the assumed displacement function for the $j$-th mode of vibration. We consider this to be the vibration mode of the cantilever without the attached object as given by equation (6). This is an approximation and this will be investigated numerically later.

The kinetic energy of the system contributes to $m_{e q_{j}}$ while the potential energy contributes to $k_{e q_{j}}$. The total kinetic energy comes from three components, namely, the kinetic energy of the cantilever, kinetic energy of the attached mass due to linear velocity and kinetic energy of the attached mass due to rotational velocity. Assuming harmonic 
motion, the overall equivalent mass $m_{e q_{j}}$ can be expressed as

$$
\begin{aligned}
m_{e q_{j}} & =\rho A L \int_{0}^{1} Y_{j}^{2}(\xi) \mathrm{d} \xi+M Y_{j}^{2}(1)+\left.J\left(\frac{\partial Y_{j}}{\partial x}\right)^{2}\right|_{\xi=1} \\
& =\rho A L \int_{0}^{1} Y_{j}^{2}(\xi) \mathrm{d} \xi+M Y_{j}^{2}(1)+\frac{J}{L^{2}} Y_{j}^{\prime 2}(1) \\
& =\rho A L[\underbrace{\int_{0}^{1} Y_{j}^{2}(\xi) \mathrm{d} \xi}_{I_{1}}+\alpha Y_{j}^{2}(1)+\beta Y_{j}^{\prime 2}(1)]
\end{aligned}
$$

From the potential energy, the equivalent stiffness $k_{e q_{j}}$ can be obtained as

$$
k_{e q_{j}}=\frac{E I}{L^{3}} \underbrace{\int_{0}^{1} Y_{j}^{\prime \prime 2}(\xi) \mathrm{d} \xi}_{I_{2}}
$$

From these expressions we have

$$
\frac{k_{e q_{j}}}{m_{e q_{j}}}=\left(\frac{E I}{\rho A L^{4}}\right) \frac{I_{2}}{I_{1}+\alpha Y_{j}^{2}(1)+\beta Y_{j}^{\prime 2}(1)}
$$

Using the expression of the natural frequency we have

$$
f_{j}=\frac{1}{2 \pi} \sqrt{\frac{k_{e q_{j}}}{m_{e q_{j}}}}=\frac{c_{0}}{2 \pi} \frac{\gamma_{1_{j}}}{\sqrt{1+\gamma_{2_{j}} \alpha+\gamma_{j} \beta}}, \quad j=1,2,3, \cdots
$$

The mode dependent constants can be evaluated exactly as

$$
\begin{aligned}
\gamma_{1_{j}} & =\sqrt{\frac{I_{2}}{I_{1}}}=\sqrt{\frac{\int_{0}^{1} Y_{j}^{\prime \prime 2}(\xi) \mathrm{d} \xi}{\int_{0}^{1} Y_{j}^{2}(\xi) \mathrm{d} \xi}}=\lambda_{j}^{2} \\
\gamma_{2_{j}} & =\frac{Y_{j}^{2}(1)}{I_{1}}=\frac{Y_{j}^{2}(1)}{\int_{0}^{1} Y_{j}^{2}(\xi) \mathrm{d} \xi}=4(\text { for all } j) \\
\text { and } \quad \gamma_{j} & =\frac{Y_{j}^{\prime 2}(1)}{I_{1}}=\frac{Y_{j}^{\prime 2}(1)}{\int_{0}^{1} Y_{j}^{2}(\xi) \mathrm{d} \xi}
\end{aligned}
$$

The expression of the natural frequency given by equation (37) is valid for any mode number. In view of the above expressions we have

$$
f_{j}=\frac{1}{2 \pi} \sqrt{\frac{k_{e q_{j}}}{m_{e q_{j}}}}=\frac{c_{0}}{2 \pi} \frac{\lambda_{j}^{2}}{\sqrt{1+4 \alpha+\gamma_{j} \beta}}, \quad j=1,2,3, \cdots
$$

Since we have two parameters to identify, only the first two modes are necessary. Using the expression of $Y_{j}$ in equation (6) and considering that $\lambda_{1}=1.8751$ and $\lambda_{2}=4.6941$, we have $\gamma_{1}=7.579069394$ and $\gamma_{2}=91.42336885$. This completely defines the first two natural frequency of the system. 


\section{Sensor equations for simultaneous mass and rotary inertia identification}

The aim of this section is to derive explicit equations to identify $\alpha$ and $\beta$ from the first two frequency shifts. Combining equation (2) and (39) the relationship between the resonance frequencies with and without the attached mass can be obtained as

$$
f_{j}=\frac{f_{0_{j}}}{\sqrt{1+4 \alpha+\gamma_{j} \beta}}
$$

The frequency-shift can be expressed using Eq. (40) as

$$
\Delta f_{j}=f_{0_{j}}-f_{j}=f_{0_{j}}-\frac{f_{0_{j}}}{\sqrt{1+4 \alpha+\gamma_{j} \beta}}
$$

From this we can obtain the relative frequency shift as

$$
\delta_{j}=\left(\frac{\Delta f_{j}}{f_{0_{j}}}\right)=1-\frac{1}{\sqrt{1+4 \alpha+\gamma_{j} \beta}}
$$

Rearranging gives the expression

$$
\frac{1}{\sqrt{1+4 \alpha+\gamma_{j} \beta}}=\left(1-\delta_{j}\right) \quad \text { or } \quad\left(1+4 \alpha+\gamma_{j} \beta\right)=\frac{1}{\left(1-\delta_{j}\right)^{2}}, \quad j=1,2
$$

These two equations arising for two values of $j$ completely relate the change in mass and rotary inertia with the two relative frequency-shifts. Solving these equations and after some simplifications we have

$$
\begin{aligned}
\beta & =\frac{\left(2-\delta_{1}-\delta_{2}\right)\left(\delta_{2}-\delta_{1}\right)}{\left(1-\delta_{1}\right)^{2}\left(1-\delta_{2}\right)^{2}\left(\gamma_{2}-\gamma_{1}\right)} \\
\text { and } \alpha & =\frac{1}{4}\left[\frac{1}{\left(1-\delta_{1}\right)^{2}}-1-\gamma_{1} \beta\right]
\end{aligned}
$$

These are the general equations which completely relate the added mass and rotary inertia and the frequency shifts. Note from equation (45) that the value of the identified mass is affected by rotary inertia. In the special case, when the rotary inertia is neglected, substituting $\beta=0$, from equation (45) we have

$$
\alpha=\frac{1}{4}\left[\frac{1}{\left(1-\delta_{1}\right)^{2}}-1\right]
$$

This matches exactly with the previous derivations $[6,8]$. Considering the relative frequencyshift is small and expanding the right-hand side of the preceding equation in a Taylor series and keeping only the linear term, we have

$$
\alpha \approx \frac{\delta_{1}}{2} \quad \text { or } \quad \frac{M}{\rho A L} \approx \frac{1}{2}\left(\frac{\Delta f_{1}}{f_{0_{1}}}\right)
$$

This is the widely-used classical relationship between the added tip mass and the frequencyshift. From tis derivation it is clear that this relationship only holds if the rotary inertia of the mass is negligible and the frequency-shift is small compared to the frequency of the bare cantilever oscillator. 


\section{Numerical results and discussions}

\subsection{Numerical simulation of the nanomechanical sensor}

We consider a single walled carbon nanotube in cantilever configuration as shown in Fig. 1 to investigate the possibility of applying the sensor theory developed in this paper. The added mass is assumed to be DeOxy Thymidine, a nucleotide that is found in DNA. A zigzag $(7,0)$ SWCNT with Young's modulus $E=1.0 \mathrm{TPa}, L=20 \mathrm{~nm}$, density $\rho=9.517 \times 10^{3} \mathrm{~kg} / \mathrm{m}^{3}$ and thickness $t=0.08 \mathrm{~nm}$ is taken from [43]. An illustrative diagram of the system considered is shown in Fig. 2
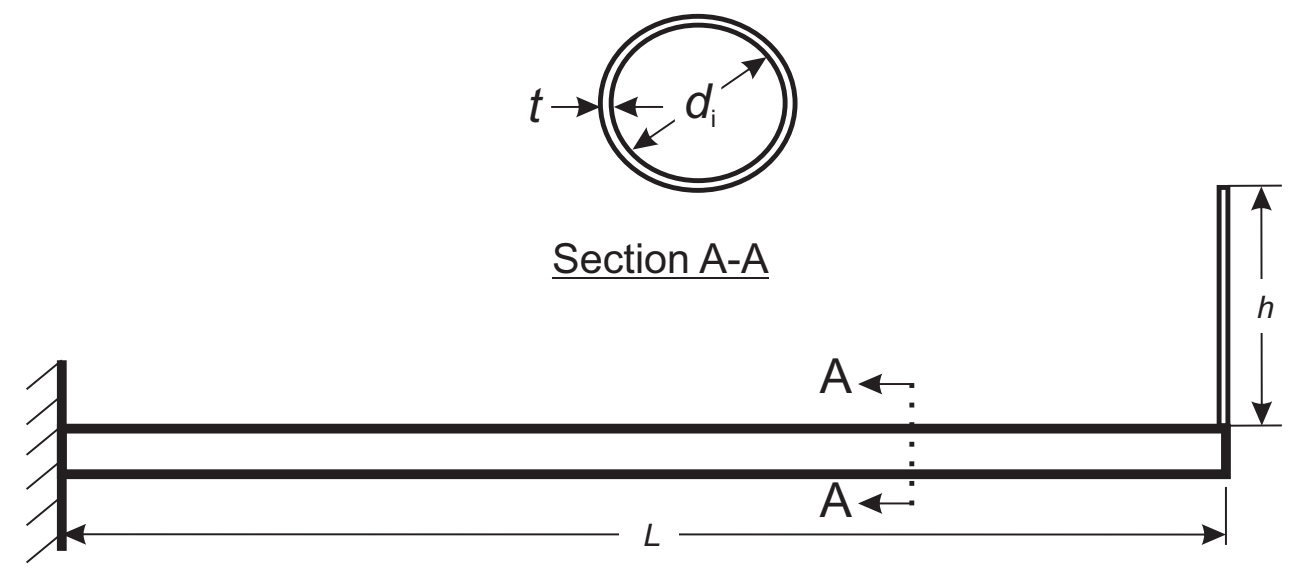

Fig. 2: illustrative diagram of a zigzag $(7,0)$ single-walled carbon nanotube (SWCNT) with clamped-free boundary condition.

For a carbon nanotube with chirality $\left(n_{i}, m_{i}\right)$, the diameter can be given by

$$
d_{i}=\frac{r}{\pi} \sqrt{n_{i}^{2}+m_{i}^{2}+n_{i} m_{i}}
$$

where $r=0.246 \mathrm{~nm}$. The diameter of the SWCNT is $0.55 \mathrm{~nm}$. Using these, the crosssectional area $A$ and area moment of inertia $I$ can be obtained as

$$
A \approx \pi d_{i} t \quad \text { and } \quad I \approx \frac{\pi}{8} d_{i}^{3} t
$$

To consider realistic values of the rotary inertia, we assume that the attached mass is a straight vertical linear object of height $h$. The mass moment of inertia of such an object is given by

$$
J=M h^{2} / 3
$$

Therefore

$$
\beta=\frac{J}{\rho A L^{3}}=\frac{M h^{2} / 3}{\rho A L^{3}}=\frac{M}{\rho A L} \frac{1}{3}\left(\frac{h}{L}\right)^{2}=\frac{\alpha}{3}\left(\frac{h}{L}\right)^{2}
$$

This implies that for physically realistic objects, $\alpha$ and $\beta$ are not independent quantities. If the mass is assumed to be a 'point mass', then $h=0$ and consequently $\beta=0$. This is an assumption commonly employed in the derivation of analytical equation governing nano-mechanical sensors. In Fig. 3 the effect of neglecting the rotary inertia on the accuracy of calculating the first two natural frequencies is shown. Four values of the $h / L$ 

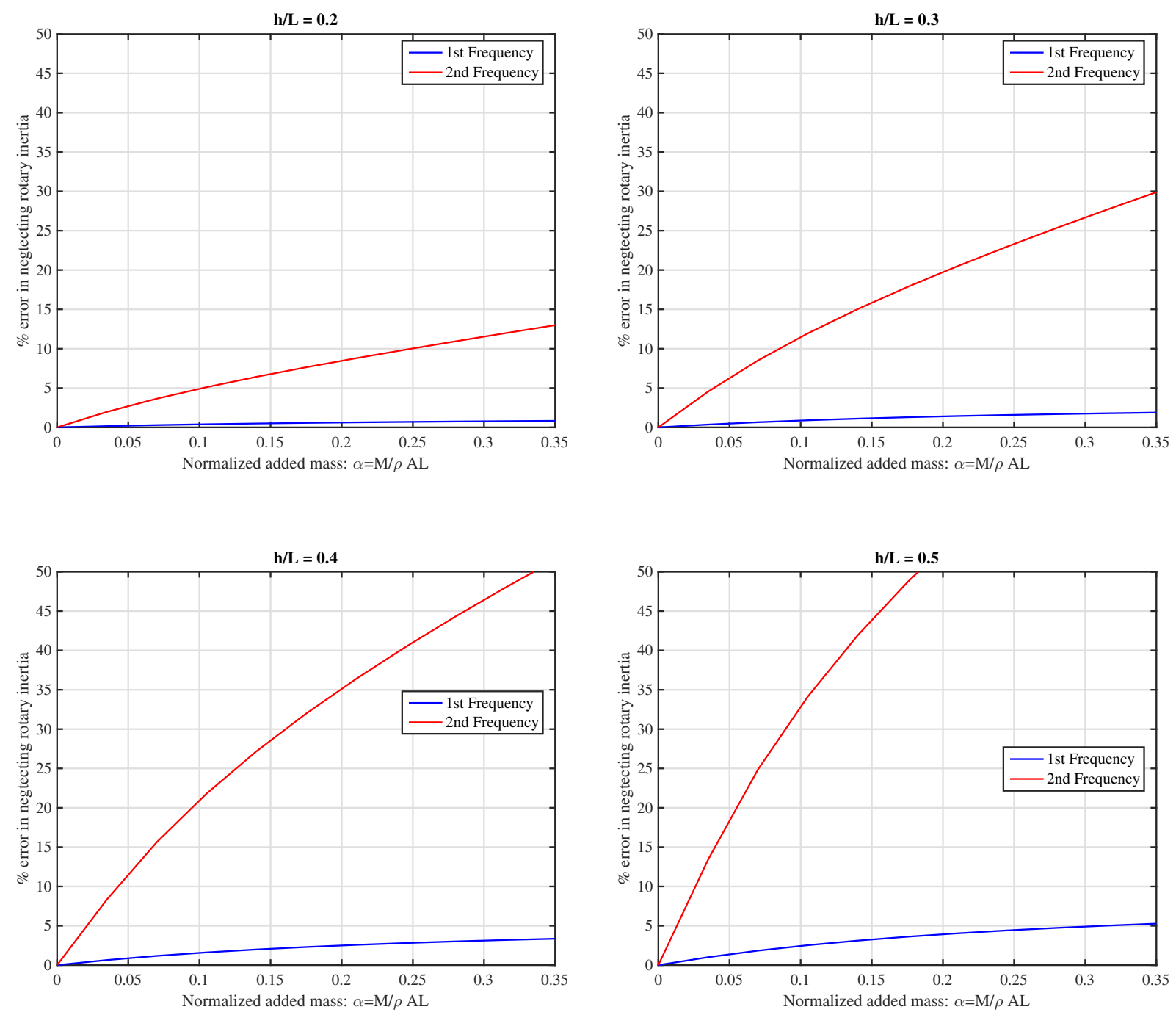

Fig. 3: Error in the first two natural frequencies arising due to neglecting the rotary inertia effect of the attached mass in cantilever nanomechanical sensor. Four values of the $h / L$ ratio are considered.

ratio, namely $0.2,0.3,0.4$ and 0.5 are selected. Frequencies for $\beta \neq 0$ are obtained by numerically solving equation (28), while those for $\beta=0$ are obtained by numerically solving equation (30). For larger values of the $h / L$ ratio, significant error in the natural frequencies is observed. For example, for $h / L=0.4$, up to $4 \%$ and $50 \%$ error in the first and second natural frequencies can be observed. This implies that the rotary inertia effect cannot be ignored for such cases.

Sensor equations proposed here is crucially based on the approximate frequency equation (39). It is important to understand any error is arising due to the use of equation (39) compared to the exact results provided by numerical solution of equation (28). The first two natural frequencies obtained using exact analytical formulation and proposed approximate equation (39) is shown in Fig. 4. The first natural frequency is approximated very well by the analytical expressions for all values of the mass ratio and $h / L$ ratio. However, discrepancies can be observed in the second natural frequency for higher values of the mass ratio and lower values of $h / L$ ratio. 

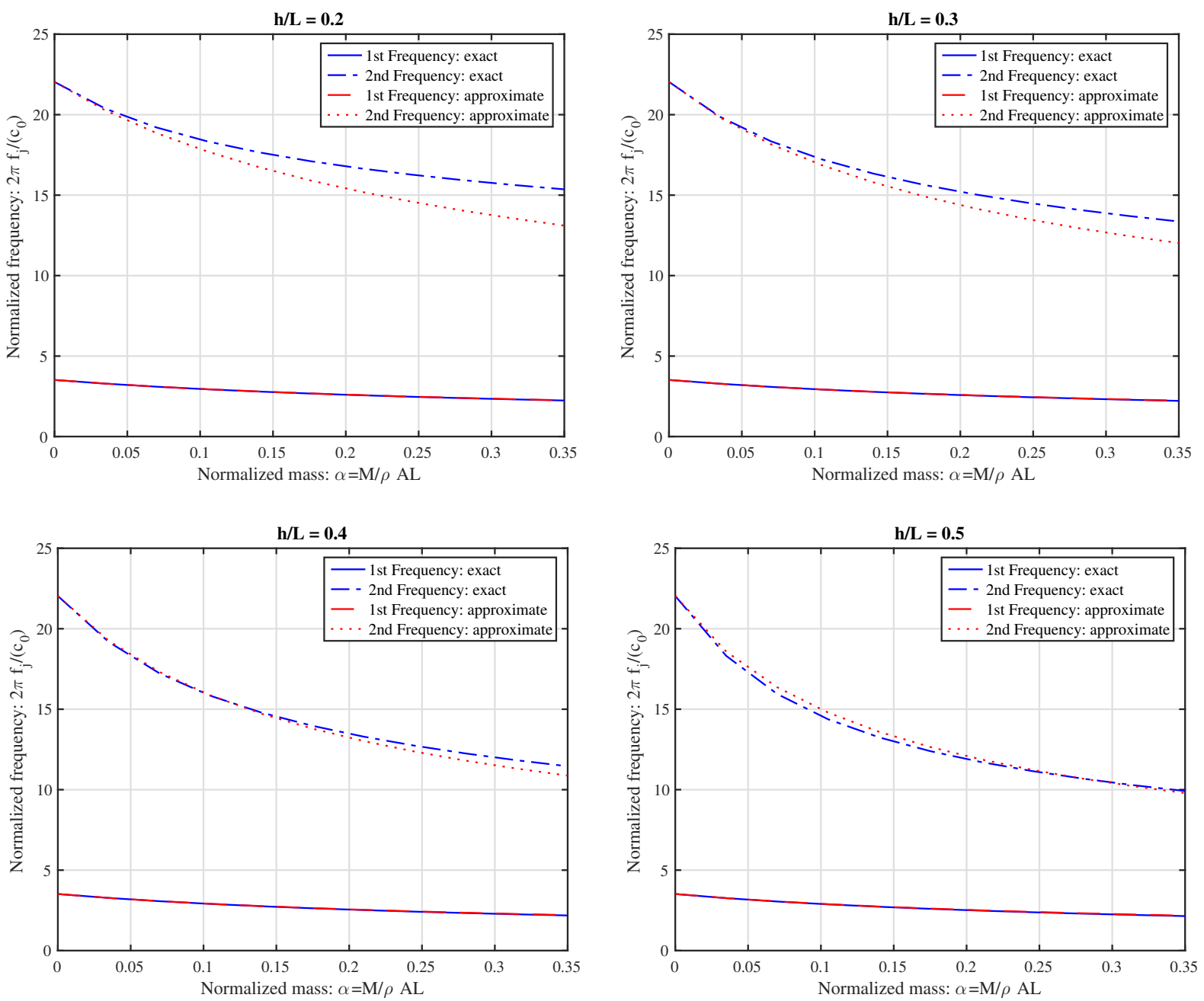

Fig. 4: First two natural frequencies obtained using exact analytical formulation and proposed approximate equation. Four values of the $h / L$ ratio are considered.

\subsection{Validation of the sensor equations}

Equations (44) and (45) give closed-form expression to detect added mass and rotary inertia from the first two frequency shifts. Consider that the frequency shifts corresponding to the two modes, namely

$$
\delta_{1}=\left(\frac{\Delta f_{1}}{f_{0_{1}}}\right)=\left(\frac{f_{0_{1}}-f_{1}}{f_{0_{1}}}\right) \quad \text { and } \quad \delta_{2}=\left(\frac{\Delta f_{2}}{f_{0_{2}}}\right)=\left(\frac{f_{0_{2}}-f_{2}}{f_{0_{2}}}\right)
$$

are available from experiment. These quantities can then be used as an 'input' to equations (44) and (45) to identify the added mass and rotary inertia. In the absence of experimental results, in this work we validate the approximate sensor equation against numerical results obtained from an independent and detailed finite element model.

The Finite element method (FEM) is a powerful numerical technique for solving partial differential equations with general boundary conditions [44]. There are broadly two approaches to model carbon nanotubes using the FEM, namely the continuum approach (see for example [45]) and the discrete approach (see for example [46]). We refer to a 


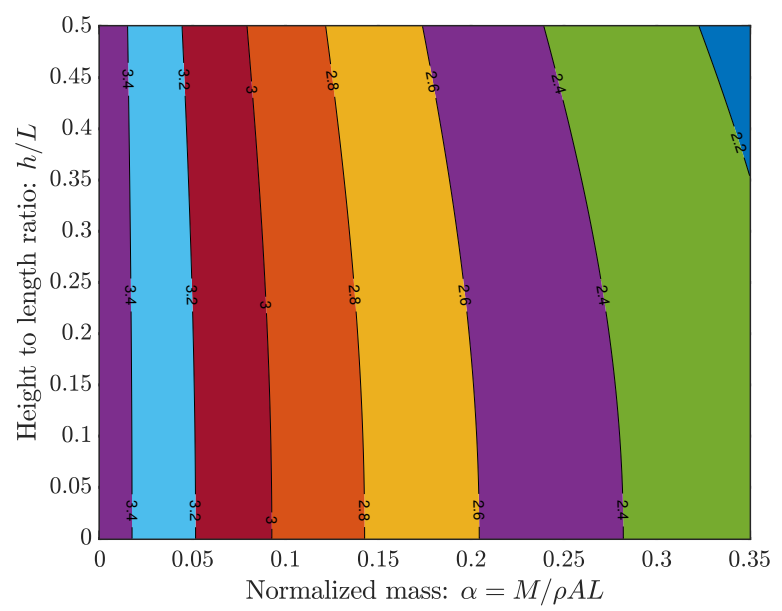

(a) Normalised first natural frequency: $2 \pi f_{1} / c_{0}$

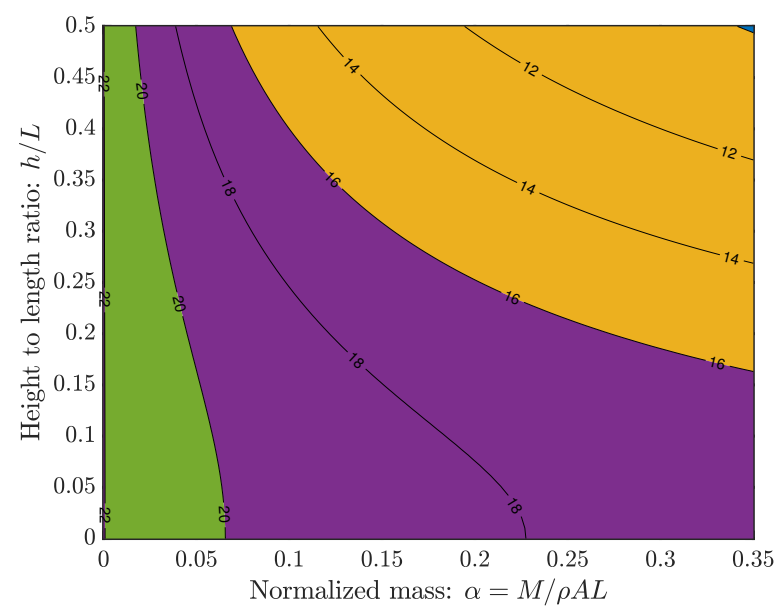

(b) Normalised second natural frequency: $2 \pi f_{2} / c_{0}$

Fig. 5: First two natural frequencies obtained using the finite element analysis. The contours of the normalised frequency values are shown as functions of normalised added mass $\alpha$ and height to length $(h / L)$ ratio. Note that first two normalised frequencies without the added mass (that is, when $\alpha=h / L=0$ ) are 3.5160 and 22.0345 respectively.

recent state-of-the-art review paper for comprehensive discussions on FEM for nanomechanical systems [47]. Here we use the continuum approach to model carbon nanotubes with attached mass and rotational inertia. Finite element model of a single-walled carbon nanotube with attached DeOxy Thymidine is implemented using the Timoshenko beam element. A convergence study on mesh size shows that element size is small enough to predict the converging results on natural frequencies and modes shapes of the model. The beam model has a circular cross-sectional area shown in Fig. 2. The geometric and material properties are taken from reference [43]. The attached DeOxy Thymidine is modelled using a Lumped mass element. The mass and moment of inertia have been defined as inertial properties of this element. In Fig. 5 we show the normalised first and second natural frequencies obtained from the finite element simulation. The frequency values are normalised as: $2 \pi f_{j} / c_{0}, j=1,2$ and the constant $c_{o}$ is defined in Eq. (4). Note that the rotary inertia parameter $\beta$ is not independent of the mass parameter $\alpha$ for this study. They are related through Eq. (51) with the height to length $(h / L)$ ratio of the attached mass. For this reason, contours of the normalised frequency values are shown as functions of $\alpha$ and $(h / L)$ in Fig. 5. The nominal values of the first two normalised frequencies without the added mass (that is when $\alpha=h / L=0$ ) are 3.5160 and 22.0345 respectively. We can observe from Fig. 5 that both frequency values reduce with increasing values of $\alpha$ and $(h / L)$. However, observe that the pattern of the change is significantly different between Figs. 5(a) and 5(b). It is this difference that is being exploited in the sensing approach proposed in this paper.

Fig. 6 compares the identified normalised mass using Equation (45) against the original mass used in the simulation. On the other hand, Fig. 7 compares the identified normalised rotary inertia using Equation (44) against the original rotary inertia used in the simulation. The value of the attached mass used is up to 0.35 times the mass of the beam. Again, four values of the $h / L$ ratio, namely $0.2,0.3,0.4$ and 0.5 are selected. 

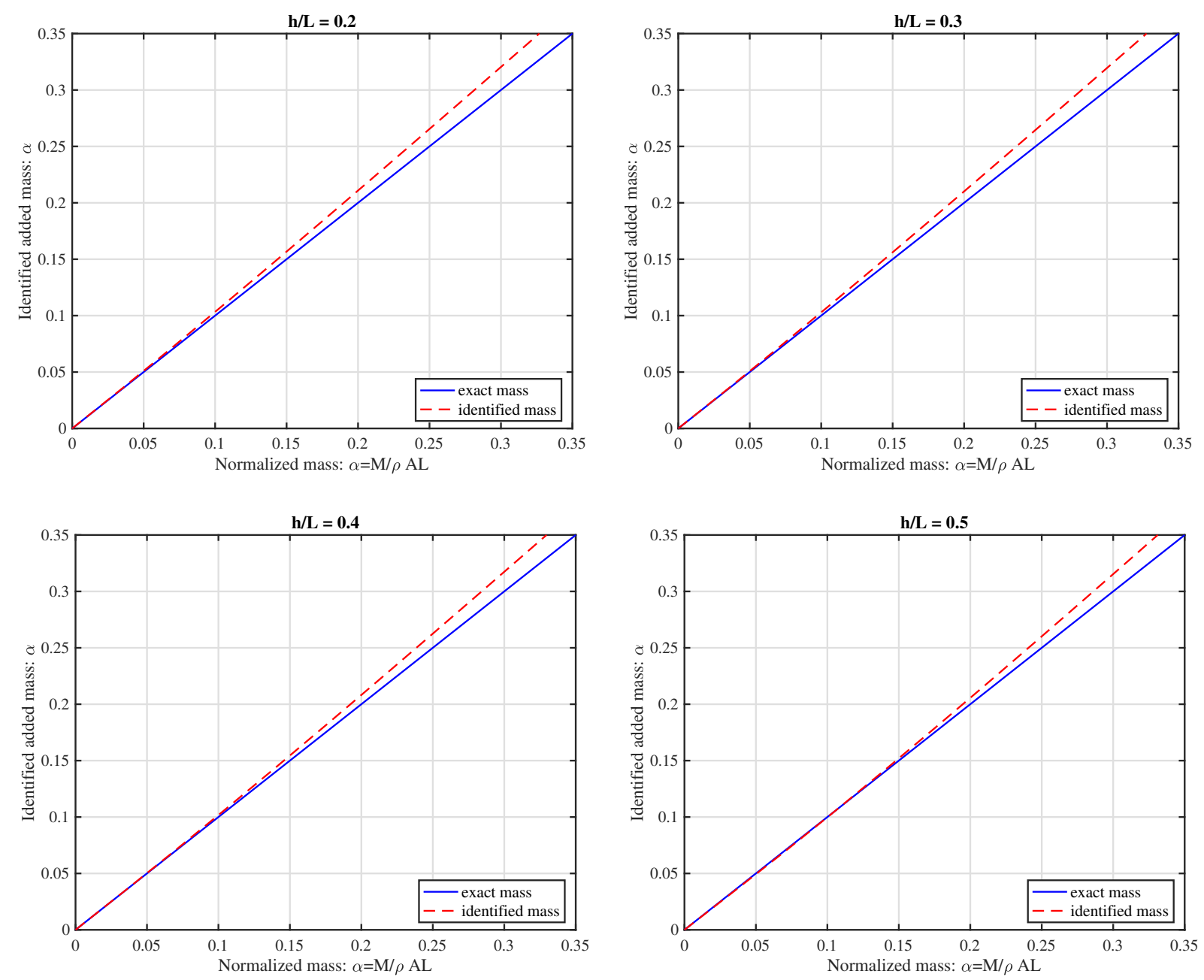

Fig. 6: Comparison between the exact attached mass and identified attached mass obtained using the proposed equation for four different height to length $(h / L)$ ratios.

The value of the identified mass is generally accurate for all values of $h / L$ ratio except for higher values of the mass ratio. The value of the identified rotary inertia is more inaccurate compared to that of the identified mass. More inaccuracies are observed for higher values of the mass ratio and lower values of the $h / L$ ratio. This is probably due to the fact that, the rotary inertia is very small for the smaller values of the $h / L$ ratio and consequently the frequency-shift is not very sensitive to the rotary inertia for this case.

\section{Conclusions}

The sensing of mass and rotary inertia of an attached object in the context of cantilever nano-mechanical sensors has been considered. Using Euler-Bernoulli cantilever beam theory, the exact equation governing the natural frequencies of the sensor with the attached mass and its rotary inertia effect has been derived. The transcendental nature of this equation does not allow the closed-form solution of the system natural frequencies. Therefore, using an energy approach, simple closed-form expressions for the identified mass and rotary inertia from the first two frequency shifts have been derived. The novel 

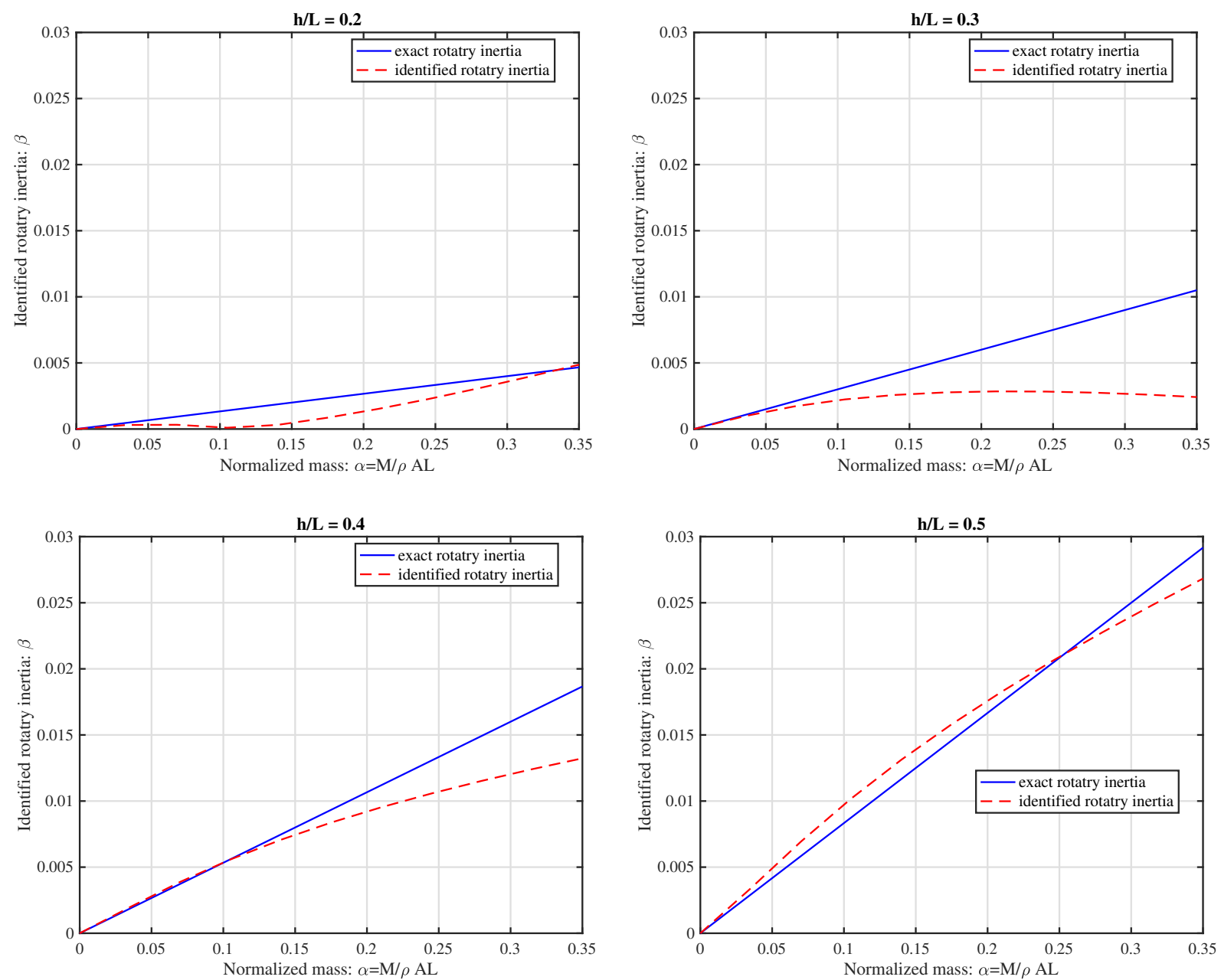

Fig. 7: Comparison between the exact rotary inertia of the attached mass and identified rotary inertia obtained using the proposed equation for four different height to length $(h / L)$ ratios.

concept introduced in this paper is the use of shifts in two resonance frequencies together, compared to the classical approach of using only one frequency shift. It was proved that the classical equation to obtain the attached mass from the first frequency shift is a special case of the general equations derived in this paper. The accuracy of the approximate sensor equations is verified using the results obtained from the solution of the exact transcendental frequency equation. Some of the highlights of this paper are:

- The prediction of the second natural frequency can be inaccurate if the rotary inertial effect is completely ignored. Therefore, it should be taken into account following the proposed approach.

- The proposed approximate closed-form expressions for both the natural frequencies give acceptable numerical accuracy when compared to the exact analytical solutions.

- The pattern of change of fist two natural frequencies of the cantilever sensor is very different due to changes in the mass and rotary inertia of the attached object.

- The new sensor equations expressed in terms of the first two frequency shifts gives 
an excellent estimate for the attached mass and comparatively less accurate estimate for the rotary inertia.

Although the proposed formulation does not predict the rotary inertia as accurately as the attached mass, it is still an improvement from the existing formulations which do not give any prediction of the rotary inertia at all. Future research should consider using the sensor equations derived here with experimentally measured frequency shifts.

\section{References}

\section{References}

[1] Q. Wang, B. Arash, A review on applications of carbon nanotubes and graphenes as nano-resonator sensors, Computational Materials Science 82 (2014) 350-360.

[2] M. Aydogdu, S. Filiz, Modeling carbon nanotube-based mass sensors using axial vibration and nonlocal elasticity, Physica E: Low-dimensional Systems and Nanostructures 43 (6) (2011) 12291234.

[3] M. De Rosa, M. Lippiello, Nonlocal timoshenko frequency analysis of single-walled carbon nanotube with attached mass: An alternative hamiltonian approach, Composites Part B: Engineering 111 (2017) 409-418.

[4] R. Chowdhury, S. Adhikari, J. Mitchell, Vibrating carbon nanotube based bio-sensors, Physica E: Low-Dimensional Systems and Nanostructures 42 (2) (2009) 104-109.

[5] Z.-B. Shen, X.-F. Li, L.-P. Sheng, G.-J. Tang, Transverse vibration of nanotube-based micro-mass sensor via nonlocal timoshenko beam theory, Computational materials science 53 (1) (2012) 340-346.

[6] R. Chowdhury, S. Adhikari, Boron nitride nanotubes as zeptogram-scale bio-nano sensors: Theoretical investigations, IEEE Transactions on Nanotechnology 10 (4) (2011) 659-667.

[7] I. Mehdipour, A. Barari, G. Domairry, Application of a cantilevered swcnt with mass at the tip as a nanomechanical sensor, Computational Materials Science 50 (6) (2011) 1830-1833.

[8] S. Adhikari, R. Chowdhury, The calibration of carbon nanotube based bio-nano sensors, Journal of Applied Physics 107 (12) (2010) 124322:1-8.

[9] A. Naderi, S. Behdad, M. Fakher, S. Hosseini-Hashemi, Vibration analysis of mass nanosensors with considering the axial-flexural coupling based on the two-phase local/nonlocal elasticity, Mechanical Systems and Signal Processing 145 (2020) 106931.

[10] T. Murmu, S. Adhikari, Nonlocal frequency analysis of nanoscale biosensors, Sensors \& Actuators: A. Physical 173 (1) (2012) 41-48.

[11] Z.-B. Shen, L.-P. Sheng, X.-F. Li, G.-J. Tang, Nonlocal timoshenko beam theory for vibration of carbon nanotube-based biosensor, Physica E: Low-dimensional Systems and Nanostructures 44 (7) (2012) 1169 - 1175 .

[12] M. Fakher, S. Rahmanian, S. Hosseini-Hashemi, On the carbon nanotube mass nanosensor by integral form of nonlocal elasticity, International Journal of Mechanical Sciences 150 (2019) 445 457.

[13] K. Jensen, K. Kim, A. Zettl, An atomic-resolution nanomechanical mass sensor, Nature Nanotechnology 3 (9) (2008) 533-537.

[14] Y. Li, X. Qiu, F. Yang, X.-S. Wang, Y. Yin, Ultra-high sensitivity of super carbon-nanotube-based mass and strain sensors, Nanotechnology 19 (16).

[15] S. B. Tooski, Functionalized single wall carbon nanotube sensor in a perturbed microwave resonant cavity based toxin/pollutant gas pressure sensor, Journal of Applied Physics 107 (3) (2010) 034315.

[16] J. Wang, M. Musameh, Carbon nanotube/teflon composite electrochemical sensors and biosensors, Analytical Chemistry 75 (9) (2003) 2075-2079.

[17] S. Tsang, J. Davis, M. Green, H. Allen, O. Hill, Y. Leung, P. Sadler, Immobilization of small proteins in carbon nanotubes - high-resolution transmission electron-microscopy study and catalytic activity, Journal of the Chemical Society-Chemical Communications (17) (1995) 1803-1804.

[18] J. Davis, M. Green, H. Hill, Y. Leung, P. Sadler, J. Sloan, A. Xavier, S. Tsang, The immobilisation of proteins in carbon nanotubes, Inorganica Chimica Acta 272 (1-2) (1998) 261-266. 
[19] S. Wong, E. Joselevich, A. Woolley, C. Cheung, C. Lieber, Covalently functionalized nanotubes as nanometre-sized probes in chemistry and biology, Nature 394 (6688) (1998) 52-55.

[20] R. Baughman, C. Cui, A. Zakhidov, Z. Iqbal, J. Barisci, G. Spinks, G. Wallace, A. Mazzoldi, D. De Rossi, A. Rinzler, O. Jaschinski, S. Roth, M. Kertesz, Carbon nanotube actuators, Science 284 (5418) (1999) 1340-1344.

[21] M. Mattson, R. Haddon, A. Rao, Molecular functionalization of carbon nanotubes and use as substrates for neuronal growth, Journal of Molecular Neuroscience 14 (3) (2000) 175-182.

[22] F. Lu, L. Gu, M. J. Meziani, X. Wang, P. G. Luo, L. M. Veca, L. Cao, Y.-P. Sun, Advances in bioapplications of carbon nanotubes, Advanced Materials 21 (2) (2009) 139-152.

[23] N. Kam, M. O'Connell, J. Wisdom, H. Dai, Carbon nanotubes as multifunctional biological transporters and near-infrared agents for selective cancer cell destruction, Proceedings of the National Academy of Sciences of the United States of America 102 (33) (2005) 11600-11605.

[24] L. Lacerda, S. Raffa, M. Prato, A. Bianco, K. Kostarelos, Cell-penetrating cnts for delivery of therapeutics, Nano Today 2 (6) (2007) 38-43.

[25] M. Prato, K. Kostarelos, A. Bianco, Functionalized carbon nanotubes in drug design and discovery, Accounts of Chemical Research 41 (1) (2008) 60-68.

[26] H. Wang, L. Gu, Y. Lin, F. Lu, M. J. Meziani, P. G. Luo, W. Wang, L. Cao, Y.-P. Sun, Unique aggregation of anthrax (bacillus anthracis) spores by sugar-coated single-walled carbon nanotubes, Journal of the American Chemical Society 128 (41) (2006) 13364-13365.

[27] A. Y. Joshi, S. C. Sharma, S. Harsha, Zeptogram scale mass sensing using single walled carbon nanotube based biosensors, Sensors and Actuators A: Physical 168 (2) (2011) 275 - 280.

[28] A. Y. Joshi, S. Harsha, S. C. Sharma, Vibration signature analysis of single walled carbon nanotube based nanomechanical sensors, Physica E: Low-dimensional Systems and Nanostructures 42 (8) (2010) $2115-2123$.

[29] S. K. Georgantzinos, N. K. Anifantis, Carbon nanotube-based resonant nanomechanical sensors:a computational investigation of their behavior, Physica E-Low-Dimensional Systems \& Nanostructures 42 (5) (2010) 1795-1801.

[30] K. R. Byun, K. Lee, O. K. Kwon, Molecular dynamics simulation of cantilevered single-walled carbon nanotube resonators, Journal of Computational and Theoretical Nanoscience 6 (11, Sp. Iss. SI) (2009) 2393-2397.

[31] D. Du, M. Wang, J. Cai, Y. Qin, A. Zhang, One-step synthesis of multiwalled carbon nanotubes-gold nanocomposites for fabricating amperometric acetylcholinesterase biosensor, Sensors and Actuators B-Chemical 143 (2) (2010) 524-529.

[32] J. Davis, K. Coleman, B. Azamian, C. Bagshaw, M. Green, Chemical and biochemical sensing with modified single walled carbon nanotubes, Chemistry-A European Journal 9 (16) (2003) 3732-3739.

[33] S. Lee, D. S. Yoon, Nanomechanical resonators and their potential to biosensor applications, Biochip Journal 1 (3) (2007) 193-199.

[34] R. Chen, S. Bangsaruntip, K. Drouvalakis, N. Kam, M. Shim, Y. Li, W. Kim, P. Utz, H. Dai, Noncovalent functionalization of carbon nanotubes for highly specific electronic biosensors, Proceedings of the National Academy of Sciences of the United States of America 100 (9) (2003) 4984-4989.

[35] J. Gooding, R. Wibowo, J. Liu, W. Yang, D. Losic, S. Orbons, F. Mearns, J. Shapter, D. Hibbert, Protein electrochemistry using aligned carbon nanotube arrays, Journal of the American Chemical Society 125 (30) (2003) 9006-9007.

[36] J. Li, H. Ng, A. Cassell, W. Fan, H. Chen, Q. Ye, J. Koehne, J. Han, M. Meyyappan, Carbon nanotube nanoelectrode array for ultrasensitive dna detection, Nano Letters 3 (5) (2003) 597-602.

[37] L.-C. Jiang, W.-D. Zhang, A highly sensitive nonenzymatic glucose sensor based on cuo nanoparticles-modified carbon nanotube electrode, Biosensors \& Bioelectronics 25 (6) (2010) 1402 1407.

[38] D. Wu, W. Chien, C. Chen, H. Chen, Resonant frequency analysis of fixed-free single-walled carbon nanotube-based mass sensor, Sensors and Actuators A-Physical 126 (1) (2006) 117-121.

[39] L. Meirovitch, Principles and Techniques of Vibrations, Prentice-Hall International, Inc., New Jersey, 1997.

[40] R. D. Blevins, Formulas for Natural Frequency and Mode Shape, Krieger Publishing Company, Malabar, FL, USA, 1984. 
[41] K. F. Graff, Wave motion in elastic solids, Dover books on physics, Dover, New York, NY, 1991.

[42] Young W C, Budynas R G, Roark's Formulas for Stresses and Strains, 7th Edition, McGraw-Hill, Inc, London, 2002.

[43] T. Murmu, S. Adhikari, Nonlocal vibration of carbon nanotubes with attached buckyballs at tip, Mechanics Research Communications 38 (1) (2011) 62-67.

[44] O. C. Zienkiewicz, R. L. Taylor, The Finite Element Method, 4th Edition, McGraw-Hill, London, 1991.

[45] R. Chowdhury, C. Y. Wang, S. Adhikari, Low frequency vibration of multiwall carbon nanotubes with heterogeneous boundaries, Journal of Physics D: Applied Physics 43 (085405) (2010) 1-8.

[46] R. Chowdhury, S. Adhikari, C. Y. Wang, F. Scarpa, A molecular mechanics approach for the vibration of single walled carbon nanotubes, Computational Materials Science 48 (4) (2010) 730-735.

[47] Y. Chandra, S. Adhikari, E. I. Saavedra Flores, L. Figiel, Advances in finite element modelling of graphene and associated nanostructures, Materials Science and Engineering: R: Reports 140 (4) (2020) 100544:1-39. 